\title{
Insulin auto-antigenicity in type 1 diabetes
}

\section{Arising from: M. Nakayama et al. Nature 435, 220-223 (2005) and S. C. Kent et al. Nature 435, 224-228 (2005)}

Spontaneous type 1 diabetes occurs when the autoimmune destruction of pancreatic $\beta$-islet cells prevents production of the hormone insulin. This causes an inability to regulate glucose metabolism, which results in dangerously raised blood glucose concentrations. It is generally accepted that thymus-derived lymphocytes ( $\mathrm{T}$ cells) are critically involved in the onset and progression of type 1 diabetes, but the antigens that initiate and drive this destructive process remain poorly characterized - although several candidates have been considered ${ }^{1}$. Nakayama et al. ${ }^{2}$ and Kent et al. ${ }^{3}$ claim that insulin itself is the primary autoantigen that initiates spontaneous type 1 diabetes in mice and humans, respectively, a result that could have implications for more effective prevention and therapy. However, I believe that this proposed immunological role of insulin may be undermined by the atypical responses of $\mathrm{T}$ cells to the human insulin fragment that are described by Kent et al. ${ }^{3}$.

Nakayama and colleagues ${ }^{2}$ eliminated the normal insulin genes in a non-obese diabetic (NOD) mouse model and replaced them with an engineered gene encoding insulin that is hormonally active but fails to stimulate T-cell responses. The mice did not develop insulin antibodies, inflammation of $\beta$-islet cells (insulitis), or diabetes. This important and surprising finding indicates that the absence of an immune response to a single fragment of the insulin B-chain, residues 9-23, could be sufficient to prevent onset of a disease that might be expected to be mediated by immune reactivity to many other potential $\beta$-cell autoantigens.

How could this happen? T cells recognize, and are activated by, antigenic peptide fragments bound to gene products of the major histocompatibility complex (MHC). $\mathrm{CD}^{+} \mathrm{T}$ cells in the NOD mouse recognize the insulin B9-23 fragment complexed to the I- $\mathrm{A}^{\mathbb{g}^{7}}$ MHC class II molecule ${ }^{4}$ CD $8^{+} \mathrm{T}$ cells recognize a related fragment, $\mathrm{B} 15-23$, in association with the $\mathrm{H}-\mathrm{K}^{\mathrm{d}}$ class I MHC molecule ${ }^{5}$. The mutated insulin transgene used by Nakayama et al. encodes a molecule with a tyrosine-to-alanine replacement at residue 16: presumably this causes the B9-23 fragment to bind with insufficient avidity to this $\mathrm{I}-\mathrm{A}^{8^{7}}$ molecule and/or failure of the $\mathrm{B} 15-23$ peptide to bind to $\mathrm{H}-\mathrm{K}^{\mathrm{d}}$, making these insulin fragments unrecognizable to the T-cell-receptor repertoire. Prevention of diabetes in these NOD mice therefore involved replacing an immunogenic and hormonally active insulin molecule with a nonimmunogenic one.

Kent and colleagues ${ }^{3}$ recovered T-cell lymphocytes from the pancreatic-draining lymph nodes of diabetic patients, cloned them and attempted to identify the protein antigen fragments recognized by these $\mathrm{T}$ cells. Remarkably, a substantial proportion (about $50 \%$ ) of $\mathrm{CD}^{+} \mathrm{T}$ cells recognized the amino-terminal fragment (residues 1-15) of the insulin A-chain. More surprising, however, is that the proliferative responses by these clones to the insulin peptide were at best minimal: stimulation indices were three- to sixfold; one of three clones gave an 80 -fold increase in interleukin- 13 production, whereas the other two produced interleukin-13 in amounts that were only three to six times above background; and very large amounts of peptide ( $1 \mathrm{mM}$, or about $1.6 \mathrm{mg} \mathrm{ml}^{-1}$ ) were required to elicit these responses.

These seem to be valid $\mathrm{T}$-cell responses in that they can be blocked by monoclonal antibodies against MHC class II peptides, but they are notably atypical in the amount of antigen required for stimulation and in the level of the resulting response. For example, this does not compare with the functional avidity of most myelin-specific $\mathrm{CD} 4^{+} \mathrm{T}$ cells from patients with multiple sclerosis, which have $\mathrm{EC}_{50}$ values (where $\mathrm{EC}_{50}$ is the effective concentration of antigen that causes half-maximal response) in the range 1-50 $\mu \mathrm{M}$ (ref. 6), corresponding to an antigen concentration that is $200-1,000$ fold lower; also, a typical encephalitogenic $\mathrm{CD} 4^{+} \mathrm{T}$-cell clone in experimental allergic encephalomyelitis, an autoimmune murine model for multiple sclerosis, requires a concentration of a myelin basic protein peptide fragment in the $300 \mathrm{nM}$ range (about $300 \mathrm{ng} \mathrm{ml}^{-1}$ ) for activation ${ }^{7}$, which is 5,000 fold lower.

The results of Kent et al. may indicate either that the native ligand for these pancreatic lymph-node T-cell dones uses a sequence other than the first 15 residues of the insulin A-chain and is a different nominal antigen that may be unrelated to the autoimmune process, or that these anti-insulin dones have a verylow avidity. But in the latter case, where in the body would the required concentration of insulin peptide be available to activate these cells? Darcy B. Wilson

Torrey Pines Institute for Molecular Studies,

San Diega, California 92121-1122, USA

e-mait dbwilson@tpims.org

1. Castano, L. \&Eisenbarth, G.S. Annu Rev. Immunal. 8, 647-679 (1990).

2. Nakayama, $M$ etal. Nature 435, 220-223 (2005)

3. Kent, S C etal. Nature 435, 224-228(2005).

4. Abiru, N etal. L. Autoimmun 14, 231-237 (2000).

5. Wong F.S, Moustakas, A. K., Wen, L, Papadopoulos, G.K. \&Janeway, C. A. Ir Proc. Natl Acad. Sci. USA 99, 5551-5556 (2002).

6. Muraro, P. A, Pette, M, Blelekova, B, McFarland,H.F.\& Martin R.J. Immunal. 164, 5474-5481 (2000).

7. Kumac, V.et al. Proc. Natl Acad. Sci. USA 92, 9510-9514 (1995).

doi:101038/nature 04423

\section{IMMUNOLOGY}

\section{Kent et al. reply}

Replying to: D. B. Wilson Nature 438, doi:10.1038/nature04423(2005)

Wilson discusses the avidity of the lymphnode T-cell dones we described ${ }^{1}$ that recognize the insulin A1-15 fragment. He questions whether large enough concentrations of insulin would be available in situ for antigenic stimulation of $\mathrm{T}$ cells ${ }^{2}$, given the amount of peptide required to stimulate the clones and their degree of responsiveness.

To evaluate the response of these insulinreactive $T$ cells generated without antigen stimulation, relative to that of human autoreactive $\mathrm{T}$-cell clones generated in response to myelin basic protein (MBP), we have compared the proliferative response of the Ob.1A12 T-cell clone (which recognizes the MBP p85-99 epitope $e^{2}$ ) with that of our insulin-peptide-reactive T-cell clones (unpublished). The subject was
$D R 2$ - and DR4-positive and the clone was generated using autologous antigen-presenting cells expressing both human leukocyte antigen (HLA) class II alleles. The response with DRB1*1501 as a restricting element was stronger, requiring an $\mathrm{EC}_{50}$ of $0.1-10 \mu \mathrm{M} \mathrm{MBP}$ p85-99 peptide, whereas the response with DRB1*0401 required an $\mathrm{EC}_{50}$ of about $100 \mu \mathrm{M}$ peptide; this was similar to the $\mathrm{EC}_{50}$ response of insulin-peptide-reactive $\mathrm{T}$ cells restricted by DRB1*0401 (ref. 1). $\left(\mathrm{EC}_{50}\right.$ is the effective concentration of antigen that causes half-maximal response.)

It is known that human T-cell clones in long-term culture (grown for years, for example) vary in their amount of proliferation and cytokine secretion (our unpublished observa- 